\title{
Recombinant DNA (rDNA)
}

National Human Genome Research Institute (NHGRI)

\section{Source}

National Human Genome Research Institute (NHGRI). Recombinant DNA (rDNA).

Recombinant DNA (rDNA) is a technology that uses enzymes to cut and paste together DNA sequences of interest. The recombined DNA sequences can be placed into vehicles called vectors that ferry the DNA into a suitable host cell where it can be copied or expressed. 